\title{
An immune stratification reveals a subset of PD-1/LAG-3 double-positive triple- negative breast cancers
}

\author{
Giulia Bottai ${ }^{1}$, Carlotta Raschioni ${ }^{1}$, Agnese Losurdo ${ }^{2}$, Luca Di Tommaso ${ }^{3}$, Corrado Tinterri ${ }^{4}$, Rosalba Torrisi ${ }^{2}$, \\ Jorge S. Reis-Filho ${ }^{5}$, Massimo Roncalli3 ${ }^{3,6}$, Christos Sotiriou', Armando Santoro ${ }^{2,6}$, Alberto Mantovani ${ }^{6,8}$, \\ Sherene Loi $^{9}$ and Libero Santarpia ${ }^{1 *}$
}

\begin{abstract}
Background: Stromal tumor-infiltrating lymphocytes (TILs) are a robust prognostic factor in triple-negative breast cancer (TNBC). However, the clinical significance of TILs may be influenced by the complex landscape of the tumor immune microenvironment. In this study, we aimed to evaluate the composition and the functionality of lymphocytic infiltration and checkpoint receptors in TNBC.

Methods: Formalin-fixed, paraffin-embedded tissues were retrospectively collected from a cohort of patients with early-stage TNBC treated with adjuvant anthracycline-based chemotherapy $(n=259)$. Results were validated in an independent cohort of patients with TNBC $(n=104)$. Stromal TILs were evaluated on hematoxylin-and-eosin-stained sections. The density of CD4+, CD8+, and FOXP3+ lymphocytes, and the expression of the immune checkpoints PD-1 and LAG-3, were assessed by immunohistochemical analysis.

Results: The presence of elevated TILs positively correlated with the density of all T cell subtypes, especially cytotoxic CD8+ lymphocytes. We showed that increasing stromal TILs assessed as a continuous variable is an independent prognostic marker of prolonged relapse-free survival and overall survival in TNBC. Among immune subpopulations, CD8+ lymphocytes are the main effectors of anti-tumor immune responses. In two independent cohorts, we found that PD-1 and LAG-3 were concurrently expressed in approximately $15 \%$ of patients with TNBC. The expression of both checkpoint receptors positively correlated with the presence of TILs, but was not significantly associated with patient outcome.
\end{abstract}

Conclusions: Overall, our data indicate that the evaluation of stromal TILs remains the most reliable immune prognostic marker in TNBC, and support the clinical evaluation of anti-PD-1/PD-L1 and anti-LAG-3 in a subset of patients with TNBC who have concurrent expression of both checkpoint receptors.

Keywords: Immune checkpoints, LAG-3, Prognosis, Triple-negative breast cancer, Tumor-infiltrating lymphocytes

\section{Background}

Triple-negative breast cancer (TNBC) is usually characterized by an aggressive phenotype, associated with an increased risk of early recurrence within 3 years after diagnosis, and poor prognosis [1]. Current treatment approaches are limited to cytotoxic chemotherapy due

\footnotetext{
*Correspondence: liberosantarpia@yahoo.it;

libero.santarpia@humanitasresearch.it

'Oncology Experimental Therapeutics, IRCCS Clinical and Research Institute

Humanitas, Via Manzoni 113, 20089 Rozzano-Milan, Italy

Full list of author information is available at the end of the article
}

to the lack of specific therapeutic targets [1]. Therefore, the identification of reliable prognostic markers and novel therapeutic targets may allow a better stratification of patients with $\mathrm{TNBC}$, and provide the rationale for investigating innovative treatment strategies.

Recent evidence indicates that the immune microenvironment plays a key role in cancer progression and response to therapies [2]. Notably, the presence of tumor-infiltrating lymphocytes (TILs) is emerging as an important predictor of outcome and response to chemotherapy in TNBC [3-7]. However, the composition of 
the tumor immune microenvironment is very heterogeneous, and the functional significance of specific immune cell subpopulations remains poorly understood. Indeed, cytotoxic CD8+ T cells have been shown to be an independent favorable prognostic factor, while studies on CD4+ T helper and forkhead box protein 3 (FOXP3) $+\mathrm{T}$ regulatory cells have shown conflicting results [8].

Furthermore, even though TILs are able to identify and eliminate malignant cells, tumors have developed multiple mechanisms to maintain an immunosuppressive microenvironment, including the upregulation of inhibitory receptors, such as programmed cell death 1 (PD-1) and lymphocyte activation gene 3 (LAG-3) [9]. Recent findings demonstrate that the expression of immune markers related to immunosuppression is enriched in triple-negative/basal-like breast cancer, and correlate with prognosis and response to chemotherapy, supporting the evaluation of immunotherapy in TNBC $[6,10-14]$. Thus, a deeper understanding of the composition and the functionality of lymphocytic infiltration could be useful to predict patients' outcome, and to select patients with TNBC who may benefit from the addition of immune checkpoint drugs to standard chemotherapeutic regimens. The aim of the present study was to evaluate the composition and the functionality of lymphocytic infiltration in early-stage TNBC.

\section{Methods}

\section{Patient cohorts and tumor samples}

Formalin-fixed, paraffin-embedded (FFPE) tissues were retrospectively collected from 259 patients who underwent surgery at Humanitas Clinical and Research Institute (Rozzano - Milan, Italy). An additional independent cohort of TNBC samples $(n=104)$ collected from Humanitas Institutes (Catania and Castellanza, Italy) was used. All patients had histologically confirmed invasive ductal TNBC, and were treated with adjuvant anthracycline-based chemotherapy. The study was approved by the ethical committee of the Humanitas Hospitals. The study was conducted according to the "reporting of tumor marker studies" (REMARK) guidelines [15]. Clinical characteristics of patients included in this study are presented in Additional file 1: Table S1.

\section{Pathologic evaluation of TILs, immunohistochemical analysis and scoring}

Histopathologic analysis of stromal lymphocytic infiltration was performed on full-face hematoxylin and eosin (HE)-stained sections according to Salgado et al. [16]. Stromal TILs were defined as the percentage of tumor stroma containing infiltrating lymphocytes. Areas of adjacent normal breast, in situ carcinoma, necrosis or fibrosis were not included in the evaluation. TILs were reported in $10 \%$ increments $[3,5]$. We defined the lymphocyte-predominant breast cancer (LPBC) as TNBC with $\geq 50 \%$ infiltration of either tumor stroma or tumor nest. A binary cutoff $\geq 20 \%$ was also used to assess its potential to identify low-risk patients with TNBC stratified by nodal status, as previously described [17].

FFPE sections $(3 \mu \mathrm{m})$ from TNBC samples were deparaffinized with xylene, rehydrated with a graded ethanol series $(100 \%, 95 \%, 70 \%)$ to distilled water according to standard immunohistochemical protocols. Specificity of staining was determined by immunohistochemistry (IHC) on a set of cultured cell pellet blocks, normal specimens, and diverse tumor tissues in the form of whole sections, processed using the same fixative and processing methods as TNBC samples tested in the study [18-20]. The optimal concentration of each antibody was established performing serial titrations on serial FFPE sections. Antigen-retrieval conditions and detection methods were also optimized for each antibody to improve sensitivity and signal-to-noise ratio. Specificity was further determined by western blotting.

Reproducibility of antibodies was assessed with IHC analysis of serial FFPE sections stained under the same conditions on different days [20]. Briefly, heat-induced antigen retrieval was performed by placing slides in TrisEDTA (pH9) or citrate (pH6) buffer for 20 minutes at $98^{\circ}$ $\mathrm{C}$ using a water bath. Tissue sections were cooled in buffer for 20 minutes before the treatment with Peroxidase Blocking Reagent (Dako) for 10 minutes. Slides were then incubated with Background Sniper (Biocare) for 20 minutes, and then with anti-CD4 (1:100, clone 4B12, Dako), anti-CD8 (1:100, clone C8/144B, Dako), antiFOXP3 (1:100, clone 236A/E7, Abcam), anti-PD-1 (1:100, clone NAT105, Abcam), and anti-LAG-3 (1:200, clone 17B4, LS Bio) primary monoclonal antibodies. After washing in PBS, DAKO Envision systems (Dako) or MACH 1 Universal HRP Polymer (BioCare), and diaminobenzidine (DAB; BioCare) were used for chromogenic immunodetection, followed by counterstaining with hematoxylin. Negative control slides without primary antibody and positive controls for each marker were used for each immunostaining run. Full details on IHC protocols are provided in Additional file 1: Table S2.

IHC scoring was carried out as previously described [21, 22]. Briefly, each section was reviewed at low magnification. Positive lymphocytes in tumor stroma were counted in three high power fields (HPF; $\times 40$; Olympus BX53), which represent the spectrum of staining seen on initial overview of the whole section, and displayed as average number of stained cells per HPF [21]. Patients were divided into two groups by the median value of CD4, CD8, and FOXP3 expression on TILs for statistical analyses. Patients with $\geq 5 \%$ of TILs expressing PD-1 or LAG-3 were considered positive [22]. 
Evaluation of TILs and IHC scoring were independently performed by two pathologists, who were blinded for patient characteristics and outcome. The mean value of two assessments was used for the current analyses. Agreement between the two pathologists was measured by calculating Cohen's kappa and the interclass correlation coefficients (ICCs). The inter-observer $\mathrm{k}$ value for the categorical parameter LPBC was 0.63. The ICCs were 0.79 for TILs assessed as a continuous variable, 0.82 for CD4, 0.84 for CD8, 0.76 for FOXP3, 0.79 for PD-1, and 0.78 for LAG-3.

\section{Statistical analysis}

Clinicopathological associations were tested using Fisher's exact test and the Mann-Whitney $U$ test for categorical and continuous data, respectively. Pearson correlation analysis was performed to evaluate the correlation between variables. Patients who developed tumor recurrence within 36 months after primary surgery were considered positive for tumor relapse, whereas patients who remained free of recurrence for the same time frame were defined as having non-relapsing tumors. Relapse-free survival (RFS) was defined as the time from surgery until the detection of distant recurrence. Overall survival (OS) was defined as the time from surgery to date of death. Patients who were alive (for OS) or recurrence-free (for RFS) were censored at date of last follow up. Survival analyses were performed by the Cox univariate proportional hazards model. For visualization purposes, Kaplan-Meier analyses were used for the survival curves test (Mantel-Cox log-rank test). Forest plots were used to visualize the results of Cox univariate analysis for RFS and OS. Multivariate Cox proportional hazard regression analysis was adjusted for relevant clinical covariates, including age at diagnosis, histologic grade, lymph node status, tumor size, and tumor stage. The likelihood ratio (LR) test was used to compare the different prognostic models. Changes in the LR values $\left(\Delta \mathrm{LR} \mathrm{X}^{2}\right)$ were used to quantitatively measure the relative amount of prognostic information of one model compared with another. All tests were two-sided and the level of statistical significance was set at $P<0.05$. Statistical analyses were performed using GraphPad Prism version 5, and StatsDirect version 3.

\section{Results}

\section{Phenotypic profiling of TILs in TNBC}

The majority of TNBC samples had lymphocytic infiltration in tumor stroma. Approximately $75 \%$ of patients with TNBC had at least $10 \%$ of stromal TILs (range 10$80 \%$ ), while only $25 \%$ had virtual absence of lymphocytes (range $0-1 \%$ ). The LPBC (TILs $\geq 50 \%$ ) phenotype was found in $10.8 \%$ of patients with TNBC.
We further explored the nature of immune infiltrates by performing IHC of the main lymphocyte subsets. Immunophenotypic characterization of lymphocyte components showed that the presence of elevated TILs was positively associated with the density of CD4+ $(r=0.347)$ and FOXP3+ $(r=0.327)$ lymphocytes, and the strongest correlation was with CD8+ T cells $(r=0.511$; Fig. 1a). These results were confirmed by analyzing an additional cohort of patients with TNBC $(n=104$; Fig. 1b, Additional file 1: Table S1). Representative images of TNBC with different degrees of TILs and distinct lymphocyte subpopulations are depicted in Fig. 1c-f.

\section{Association of TILs with clinicopathological parameters and survival in TNBC}

A lower stromal TILs content was associated with larger tumor size $(P=1.8 \mathrm{E}-02$; Additional file 1: Table S3). There were no other significant associations between the variables examined and the presence of TILs, or with different immune cell subsets in a first TNBC cohort ( $\mathrm{n}=259$; Additional file 1: Table S3). The data were confirmed in the validation cohort of TNBC $(n=104$; Additional file 1: Table S4).

The association of LPBC, continuous TIL scores, and single immune components with RFS or OS in patients with TNBC was evaluated by Cox proportional hazard regression analyses (Fig. 2, Fig. 3, and Table 1). TILs assessed as a binary variable (LPBC vs non-LPBC) were associated with both RFS (hazard ratio (HR) $=0.22$; 95\% confidence interval (CI), 005 to $0.88 ; P=3.28 \mathrm{E}-02)$ and OS (HR $=0.29 ; 95 \% \mathrm{CI}, 0.09$ to $0.93 ; P=3.73 \mathrm{E}-02)$ in TNBC in univariate analysis (Fig. 2 and Fig. 3a), but had no prognostic value on multivariate analysis (Table 1 ). However, continuous TIL scores had a significant prognostic value for RFS ( $\mathrm{HR}=0.92 ; 95 \% \mathrm{CI}, 0.82$ to $0.98 ; P$ $<1.00 \mathrm{E}-04$ ) and $\mathrm{OS}$ (HR $=0.92 ; 95 \% \mathrm{CI}, 0.89$ to $0.95 ; P<$ 1.00E-04) in TNBC (Fig. 2 and Fig. 3b). Cox multivariate analysis confirmed that TIL scores were independently associated with RFS (HR $=0.93$; $95 \% \mathrm{CI}, 0.89$ to 0.96 ; $P=1.00 \mathrm{E}-04)$ and $\mathrm{OS}(\mathrm{HR}=0.93$; 95\% CI, 0.90 to 0.95 ; $P=1.00 \mathrm{E}-04)$ in TNBC (Table 1). Furthermore, continuous TIL scores added significant prognostic information for RFS $\left(\triangle \mathrm{LR} \chi^{2}=31.35 ; P<1.00 \mathrm{E}-04\right)$ and OS $\left(\Delta \mathrm{LRX}^{2}=28.23 ; P<1.00 \mathrm{E}-04\right)$ beyond that provided by standard clinicopathological variables (Table 2).

Given that recent data suggest that a stromal TIL value $\geq 20 \%$ in early-stage TNBC could identify patients with good outcome across nodal categories, we performed Kaplan-Meier analysis to evaluate the prognostic value of this cutoff in TNBC stratified by nodal status (lymph node-negative and lymph node-positive) [17]. Overall, we found that patients with high levels of TILs ( $\geq 20 \%)$ had a better outcome compared with those 


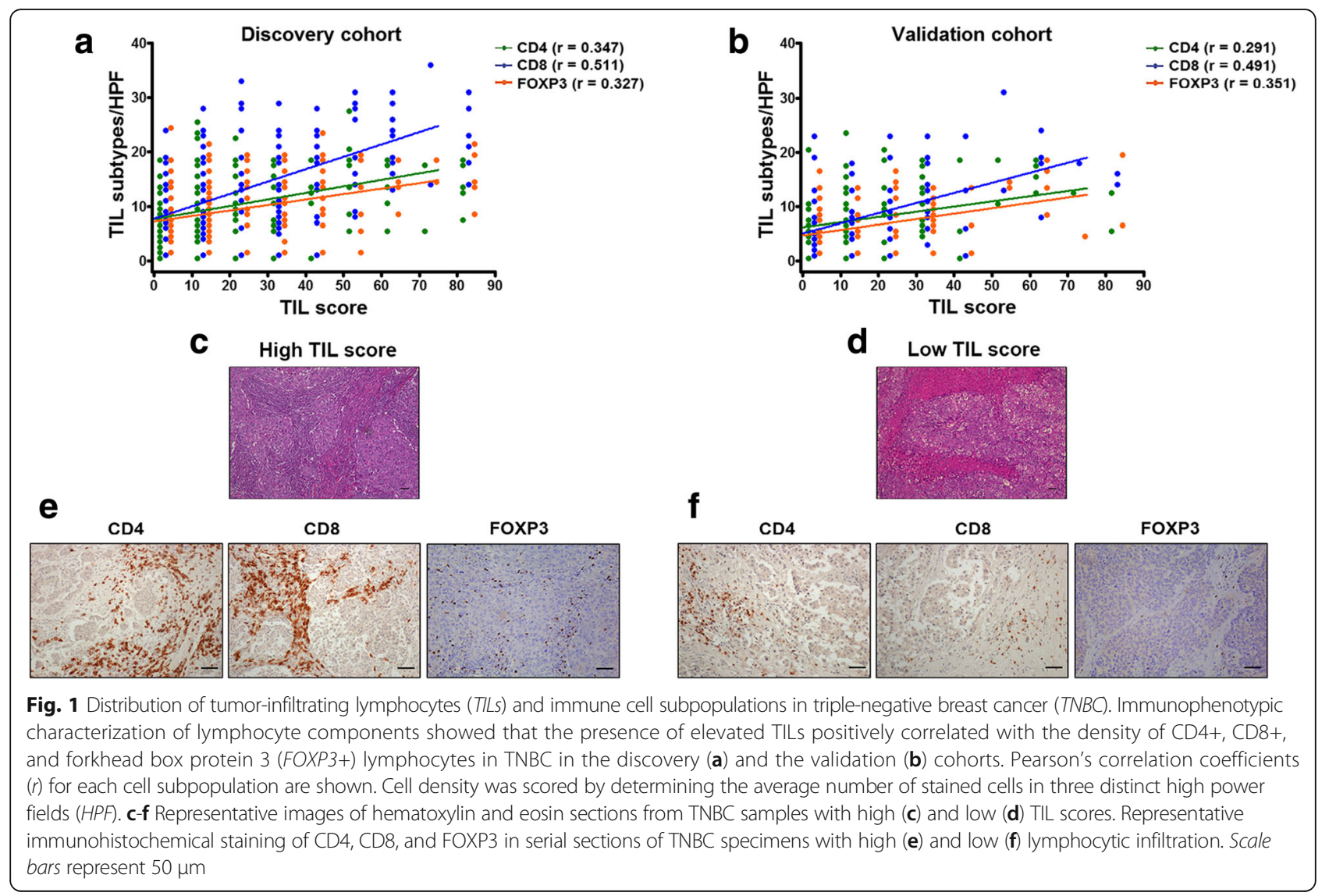

with low TILs $(<20 \%)$ in both nodal categories (Additional file 2: Figure S1).

Among lymphocyte subsets, the density of CD4+ cells was not significantly prognostic in TNBC (Fig. 2), while CD8+ lymphocytes were consistently associated with prolonged RFS and $\mathrm{OS}$ in both univariate $(\mathrm{HR}=0.54$; 95\% CI, 0.33 to $0.91 ; P=2.05 \mathrm{E}-02$ for $\mathrm{RFS}$; $\mathrm{HR}=0.54$;
95\% CI, 0.32 to $0.91 ; P=2.07 \mathrm{E}-02$ for OS) and multivariate analysis $(\mathrm{HR}=0.58$; $95 \% \mathrm{CI}, 0.34$ to $0.97 ; P=3.72 \mathrm{E}-$ 02 for RFS; $\mathrm{HR}=0.58$; $95 \% \mathrm{CI}, 0.34$ to $0.97 ; P=3.88 \mathrm{E}-02$ for OS), indicating that cytotoxic CD8+ T lymphocytes are the main effectors of anti-tumor immune responses (Fig. 2, Fig. 3c, and Table 1). Furthermore, high FOXP3+ cells were also significantly associated with better

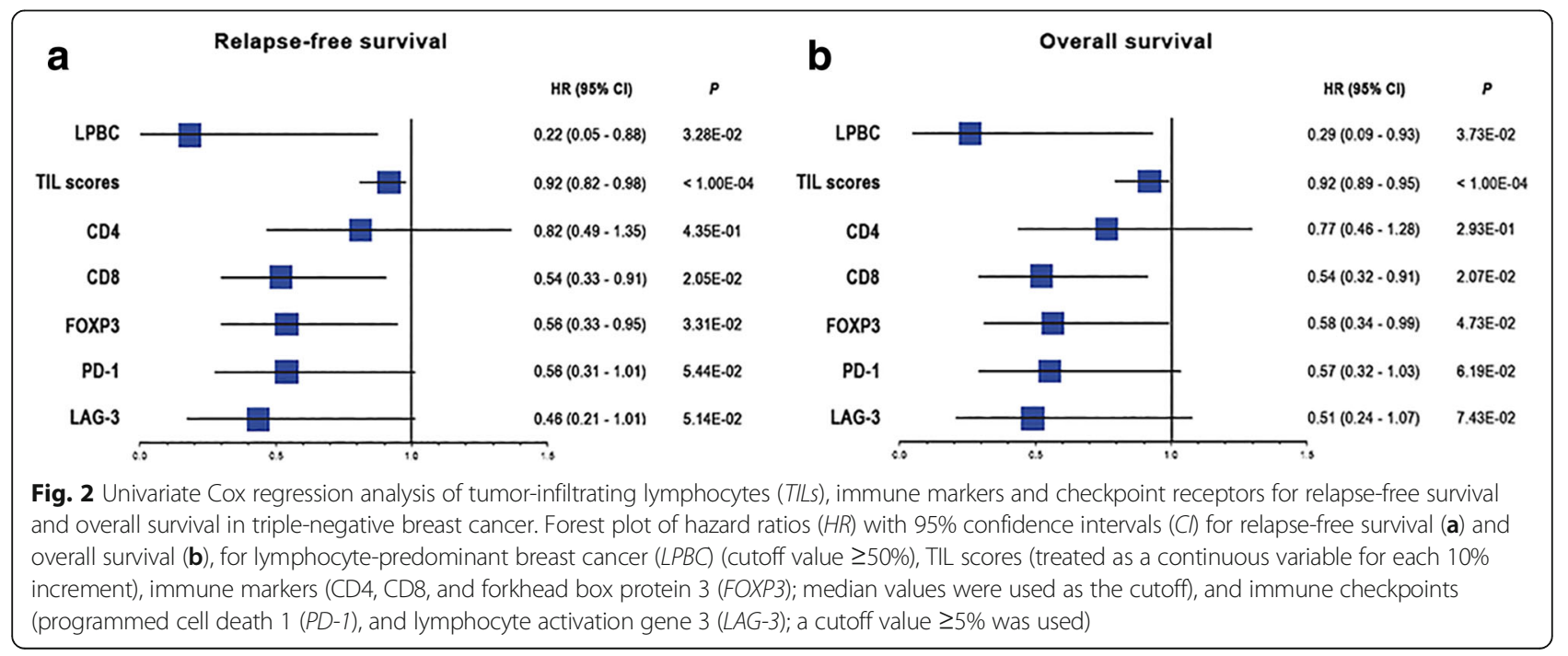




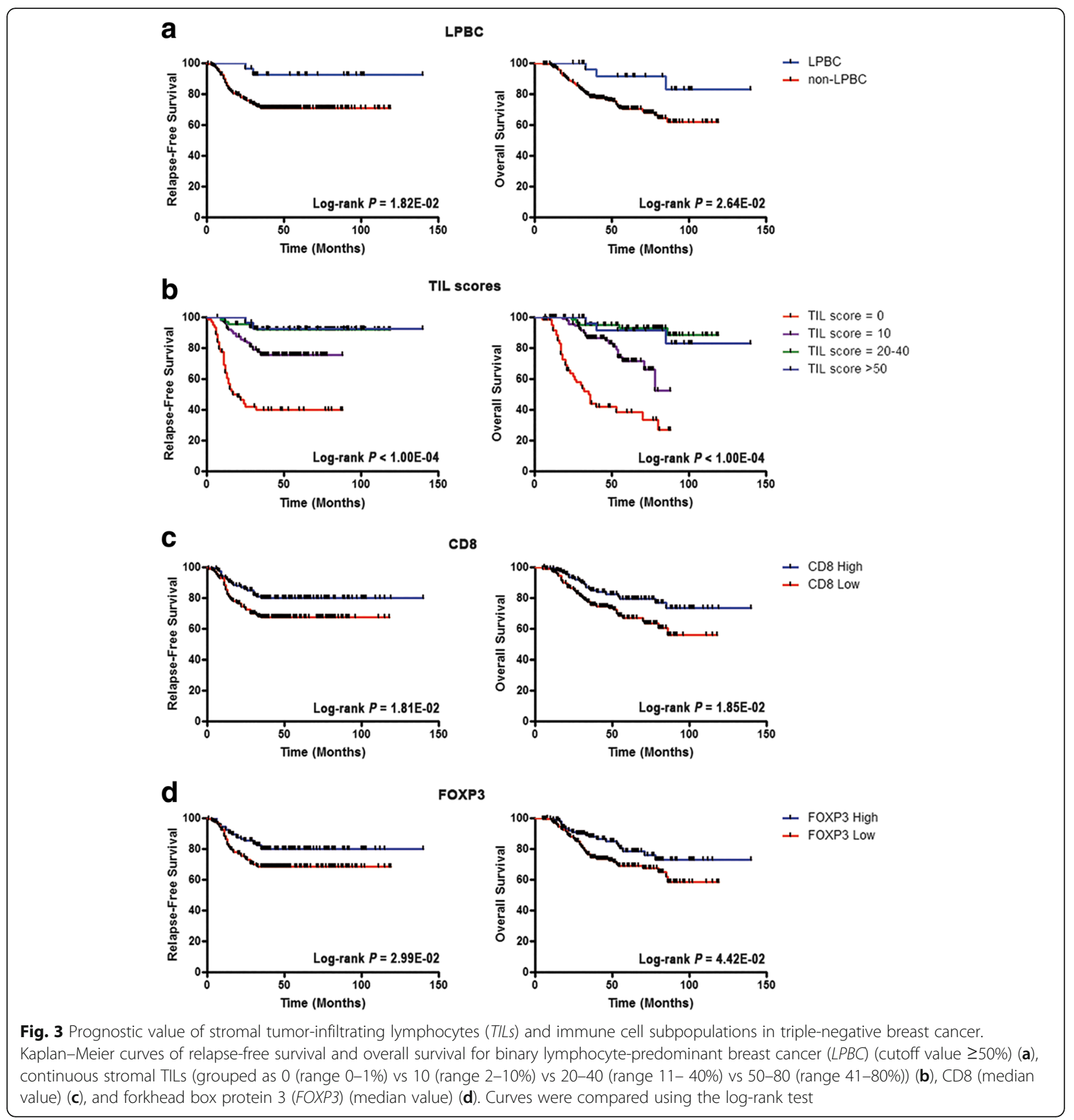

survival in univariate $(\mathrm{HR}=0.56 ; 95 \% \mathrm{CI}, 0.33$ to 0.95 ; $P=3.31$ E-02 for RFS; $\mathrm{HR}=0.58$; $95 \% \mathrm{CI}, 0.34$ to 0.99 ; $P=4.73 \mathrm{E}-02$ for $\mathrm{OS})$ and multivariate analysis $(\mathrm{HR}=0.52$; 95\% CI, 0.31 to $0.89 ; P=1.71 \mathrm{E}-02$ for RFS; $\mathrm{HR}=0.55$; $95 \%$ CI, 0.32 to $0.94 ; P=2.90 \mathrm{E}-02$ for OS; Fig. 2, Fig. 3d, and Table 1). However, FOXP3+ cells were consistently associated with the density of CD8+ lymphocytes $(r=0.716)$, and the presence of FOXP3+ TILs was prognostically insignificant in TNBC stratified by the presence or absence of CD8 + cells (Additional file 1: Table S5).
Interestingly, we found that the infiltration of FOXP3 + cells tended to be associated with reduced survival in TNBC with low numbers of CD8+ lymphocytes (Additional file 1: Table S5), suggesting that the prognostic value of FOXP3+ cells was highly dependent on the concurrent presence of cytotoxic T lymphocytes. Of note, neither CD8+ nor FOXP3+ cells added consistent prognostic value for RFS $\left(\Delta \mathrm{LR} X^{2}=0.43 ; P=5.12 \mathrm{E}-01\right.$ for CD8; $\Delta \mathrm{LR} \chi^{2}=1.02 ; P=3.12 \mathrm{E}-01$ for FOXP3) and OS $\left(\Delta \mathrm{LR} \chi^{2}=0.50 ; P=4.79 \mathrm{E}-01\right.$ for $\mathrm{CD} 8 ; \Delta \mathrm{LR}^{2}=0.99$; 
Table 1 Multivariate Cox regression analysis of tumor-infiltrating lymphocytes and immune markers for relapse-free survival and overall survival in triple-negative breast cancer

\begin{tabular}{|c|c|c|c|c|c|c|}
\hline \multirow[b]{2}{*}{ Variable } & \multicolumn{3}{|c|}{ Relapse-free survival } & \multicolumn{3}{|c|}{ Overall survival } \\
\hline & $\mathrm{HR}$ & $95 \% \mathrm{Cl}$ & $P$ value & $\mathrm{HR}$ & $95 \% \mathrm{Cl}$ & $P$ value \\
\hline$\overline{L P B C}$ & 0.24 & $0.06-1.00$ & $5.10 \mathrm{E}-02$ & 0.32 & $0.10-1.03$ & $5.60 \mathrm{E}-02$ \\
\hline Age & 1.09 & $0.66-1.80$ & $7.28 \mathrm{E}-01$ & 1.13 & $0.68-1.87$ & $6.27 \mathrm{E}-01$ \\
\hline Histologic grade & 1.66 & $0.78-3.53$ & $1.91 \mathrm{E}-01$ & 1.85 & $0.87-3.94$ & 1.09E-01 \\
\hline Nodal status & 3.44 & $1.72-6.88$ & $5.00 E-04$ & 3.19 & $1.59-6.39$ & $1.00 E-03$ \\
\hline Tumor size & 1.21 & $0.74-1.99$ & 4.48E-01 & 1.27 & $0.77-2.09$ & $3.48 \mathrm{E}-01$ \\
\hline Tumor stage & 1.34 & $0.76-2.35$ & $3.12 \mathrm{E}-01$ & 1.38 & $0.78-2.43$ & 2.69E-01 \\
\hline TILs ${ }^{a}$ & 0.93 & $0.89-0.96$ & $1.00 E-04$ & 0.93 & $0.90-0.95$ & $1.00 E-04$ \\
\hline Age & 0.97 & $0.58-1.61$ & 8.97E-01 & 1.02 & $0.61-1.71$ & $9.32 \mathrm{E}-01$ \\
\hline Histologic grade & 1.34 & $0.63-2.87$ & $4.48 \mathrm{E}-01$ & 1.76 & $0.83-3.75$ & 1.40E-01 \\
\hline Nodal status & 2.91 & $1.43-5.90$ & $3.10 E-03$ & 2.59 & $1.27-5.28$ & $8.70 E-03$ \\
\hline Tumor size & 1.11 & $0.68-1.83$ & $6.70 \mathrm{E}-01$ & 1.15 & $0.70-1.90$ & $5.73 \mathrm{E}-01$ \\
\hline Tumor stage & 1.46 & $0.82-2.62$ & $2.00 \mathrm{E}-01$ & 1.45 & $0.80-2.63$ & 2.19E-01 \\
\hline CD8 & 0.58 & $0.34-0.97$ & $3.72 E-02$ & 0.58 & $0.34-0.97$ & $3.88 E-02$ \\
\hline Age & 1.12 & $0.68-1.85$ & $6.66 \mathrm{E}-01$ & 1.16 & $0.70-1.93$ & $5.58 \mathrm{E}-01$ \\
\hline Histologic grade & 1.71 & $0.80-3.65$ & $1.67 \mathrm{E}-01$ & 1.91 & $0.90-4.08$ & $9.34 \mathrm{E}-02$ \\
\hline Nodal status & 3.46 & $1.73-6.90$ & 4.00E-04 & 3.23 & $1.62-6.45$ & $9.00 E-04$ \\
\hline Tumor size & 1.20 & $0.73-1.97$ & 4.80E-01 & 1.26 & $0.76-2.07$ & $3.70 \mathrm{E}-01$ \\
\hline Tumor stage & 1.35 & $0.76-2.37$ & $3.01 \mathrm{E}-01$ & 1.35 & $0.76-2.40$ & 2.99E-01 \\
\hline FOXP3 & 0.52 & $0.31-0.89$ & $1.71 E-02$ & 0.55 & $0.32-0.94$ & $2.90 E-02$ \\
\hline Age & 1.10 & $0.67-1.83$ & $6.98 \mathrm{E}-01$ & 1.16 & $0.70-1.92$ & $5.70 \mathrm{E}-01$ \\
\hline Histologic grade & 1.81 & $0.84-3.87$ & $1.28 \mathrm{E}-01$ & 2.01 & $0.94-4.30$ & 7.27E-02 \\
\hline Nodal status & 3.54 & $1.79-7.00$ & $3.00 E-04$ & 3.30 & $1.67-6.53$ & $6.00 E-04$ \\
\hline Tumor size & 1.23 & $0.75-2.03$ & 4.05 E-01 & 1.30 & $0.79-2.13$ & 3.06E-01 \\
\hline Tumor stage & 1.37 & $0.79-2.41$ & 2.65E-01 & 1.38 & $0.78-2.42$ & 2.66E-01 \\
\hline
\end{tabular}

Multivariate analysis adjusted for age ( $\geq 50$ vs $<5$ years), histologic grade (III vs - I-II), nodal status ( 1 vs 0 ), tumor size ( $>20 \mathrm{~mm}$ vs $\leq 20 \mathrm{~mm}$ ), and tumor stage (III vs I-II). Significant $P$ values are in italics. ${ }^{a}$ Treated as a continuous variable for each $10 \%$ increment. Cl confidence interval, $H R$ hazard ratio, $L P B C$

lymphocyte-predominant breast cancer, TILs tumor-infiltrating lymphocytes

$P=3.20 \mathrm{E}-01$ for FOXP3) beyond that provided by the TIL score in the multivariate model (Table 2), suggesting that the evaluation of single immune components may not be as informative as the global evaluation of stromal TILs.

\section{Evaluation of the clinical relevance of immune} checkpoints in TNBC

To assess the functional status of TILs in TNBC, we analyzed the expression of the checkpoint receptors PD-1 and LAG-3 by IHC. PD-1+ and LAG-3+ TILs were

Table 2 Comparisons of added prognostic information

\begin{tabular}{|c|c|c|c|c|}
\hline \multirow[b]{2}{*}{ Variable } & \multicolumn{2}{|c|}{ Relapse-free survival } & \multicolumn{2}{|c|}{ Overall survival } \\
\hline & $\Delta \mathrm{LRX}^{2}$ & $P$ value & $\Delta \mathrm{LRX^{2 }}$ & $P$ value \\
\hline $\mathrm{CP}+$ TIL score vs CP & 31.35 & $<1.00 E-04$ & 28.23 & $<1.00 E-04$ \\
\hline$C P+L P B C$ vs $C P$ & 5.20 & $2.26 E-02$ & 4.97 & $2.58 E-02$ \\
\hline$C P+$ TIL score + CD8 vs CP + TIL score & 0.43 & $5.12 \mathrm{E}-01$ & 0.50 & 4.79E-01 \\
\hline CP + TIL score + FOXP3 vs CP + TIL score & 1.02 & $3.12 \mathrm{E}-01$ & 0.99 & $3.20 \mathrm{E}-01$ \\
\hline$C P+$ TIL score $+C D 8+F O X P 3$ vs $C P+$ TIL score & 1.82 & $4.02 \mathrm{E}-01$ & 1.70 & 4.27E-01 \\
\hline
\end{tabular}

Significant $P$ values are given in italics. $C P$ clinicopathological variables (age, histologic grade, nodal status, tumor size, and tumor stage), $L P B C$ lymphocyte-predominant breast cancer, LR likelihood ratio, TILs tumor-infiltrating lymphocytes, FOXP3 forkhead box protein 3 
present in approximately $30 \%$ and $18 \%$ of patients with TNBC, respectively (Fig. 4a, b). Concurrent expression of both immune checkpoints was observed in $15.4 \%$ of TNBC cases. We found that the expression of both PD1 and LAG-3 positively correlated with the presence of TILs $(r=0.511 ; r=0.576$, respectively), particularly with CD8+ cells $(r=0.568 ; r=0.490$, respectively; Additional file 2: Figure S2a). By analyzing an additional cohort of patients with TNBC $(\mathrm{n}=104$; Additional file 1: Table S1), we confirmed that PD-1 and LAG-3 were concurrently expressed in $13.5 \%$ of patients, and that their expression was positively associated with TILs $(r=0.438$; $r=0.537$, respectively), and with CD8+ cells $(r=0.495 ; r$ $=0.467$, respectively; Additional file 2: Figure S2b). Even though a trend for longer RFS was observed in univariate analysis, the presence of both PD-1+ and LAG-3+ TILs had no significant prognostic value in the discovery dataset (Fig. 2).

\section{Discussion}

Recent evidence suggests that the presence of TILs is an important predictor of outcome and response to chemotherapy in TNBC [3-7]. In this dataset, we confirmed that increasing stromal TILs is an independent prognostic marker for prolonged RFS and OS in TNBC treated with adjuvant anthracycline-based chemotherapy. Studies evaluating the association between LPBC and survival have reported conflicting results [3-7]. Indeed, LPBC was not significantly associated with prognosis, likely due to the reduced number of events, and the small proportion of TNBC displaying this phenotype. Thus, further efforts are needed to improve the quantitative pathological assessment of TILs on HEstained slides.

In agreement with recent data, we demonstrated that a value of $\geq 20 \%$ of stromal TILs could identify a group of low-risk patients with both lymph node-negative and lymph node-positive early-stage TNBC [17]. Furthermore, our findings suggest that patients with low numbers of TILs may benefit from the generation of an anti-tumor immune response, while boosting the lymphocyte activity (e.g. checkpoint inhibitors) might prove useful in patients with high numbers of TILs, associated with higher disease burden.

Although the presence of TILs reflects the activation of a local anti-tumor immune response, distinct immune cell subpopulations may have specific biological significance. In agreement with previous findings, we demonstrated that both $\mathrm{CD} 8+$ and FOXP3+ cells were associated with good outcome in patients with TNBC, and that the clinical significance of FOXP3+ lymphocytes was highly dependent on the concurrent presence of cytotoxic T cells [23-27]. Interestingly, when stratified based on the presence of CD8+ lymphocytes, a high infiltration of FOXP3+ cells trended towards reduced survival in TNBC patients with low numbers of CD8+ cells. These results suggest that CD8+ lymphocytes could be the main effectors of anti-tumor immune responses, and that the consistent correlation between FOXP3 positivity and cytotoxic lymphocytes may in part explain conflicting results reported in previous studies [8].

Overall, our findings indicate that the assessment of single immune components may not be as informative as the global evaluation of stromal TILs. However, the
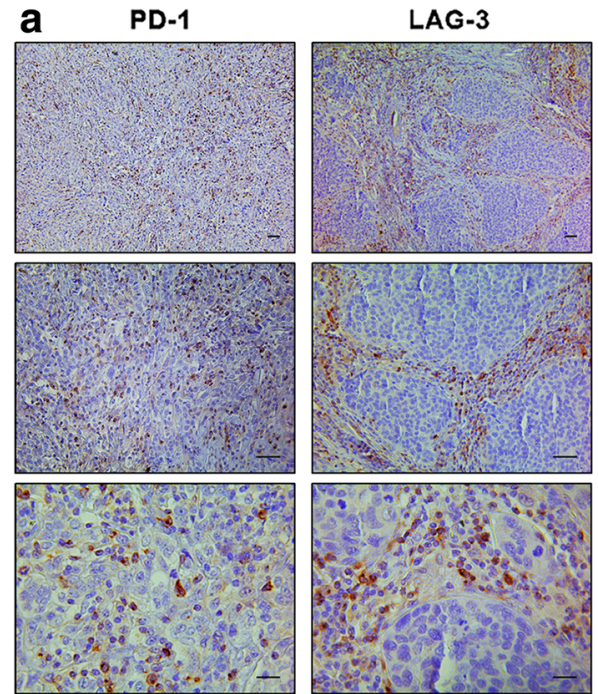
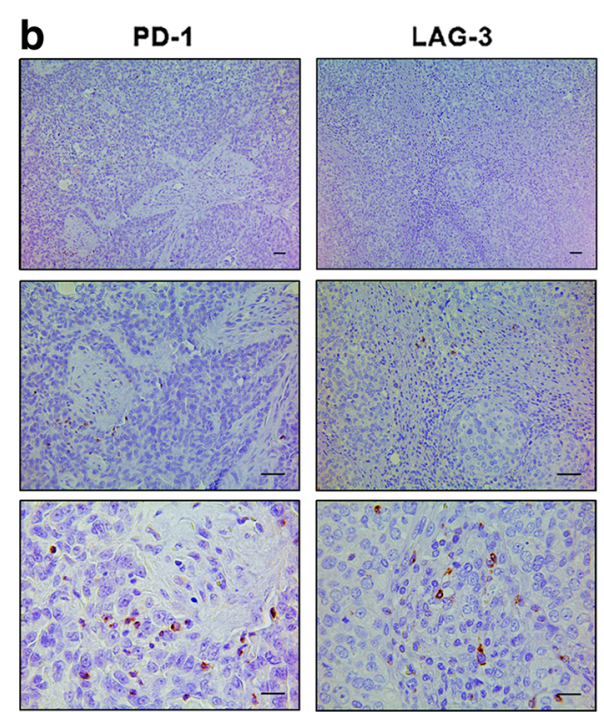

Fig. 4 Programmed cell death 1 (PD-1) and lymphocyte activation gene 3 (LAG-3) protein expression in triple-negative breast cancer (TNBC) Representative immunohistochemical staining of PD-1 and LAG-3 in serial sections of TNBC samples with high (a) and low (b) lymphocytic infiltration. Scale bars represent $50 \mu \mathrm{m}$ 
understanding of the biological role of different lymphocyte subpopulations warrants further investigations, and could be useful in selecting patients with TNBC who may benefit from the addition of specific immunomodulatory therapies to conventional chemotherapeutic regimens.

Even though TILs are emerging as important prognostic and predictive factors in TNBC, it is worth noting that many TNBC have few TILs, and even in the presence of massive lymphocytic infiltration, immunosuppressive mechanisms should be considered [28]. In this scenario, both radiotherapy and chemotherapeutic agents (e.g. anthracyclines) have been shown in preclinical models to be able to shape the tumor microenvironment, and to boost an effective immune response against tumor cells $[29,30]$. These therapies could be rationally evaluated in combination with immunomodulatory drugs to synergize with pre-existing lymphocytes with tumoricidal activity, or to elicit a de novo local immune response in tumors lacking TILs.

Even though the immune system can recognize and eliminate malignant cells, tumors have developed multiple mechanisms to evade effective immunosurveillance, including the activation of the immune checkpoints PD1 and LAG-3 [2, 9]. We demonstrated that PD-1+ and LAG-3+ TILs were present in approximately $30 \%$ and $18 \%$ of TNBCs, respectively, and that their presence in the tumor microenvironment tended to be associated with good prognosis in TNBC. The upregulation of these receptors, especially PD-1, has been classically described as a prominent immune resistance mechanism, and analyses performed on tissue microarrays have revealed an inverse correlation with outcome in patients with breast cancer $[9,31]$. Indeed, double-positive PD-1/ LAG-3 TILs have been recently demonstrated to show a more exhausted phenotype and functionality compared with single-positive or negative TILs in a preclinical model, likely leading to increased cancer immune evasion [32]. However, the role of co-inhibitory molecules in the modulation of the tumor immune microenvironment, and the mechanisms underlying $\mathrm{T}$ cell exhaustion and anergy are still poorly understood $[9,33]$. Furthermore, it is worth noting that the activity of immune cells depends on the interaction with cancer cells, and recent findings support the idea that the functional relevance of checkpoint proteins is highly sensitive to the context (e.g. amount of antigen, topographical relationships with tumor cells and PD-L1-expressing cells) [9, 34]. Consequently, the evaluation of the clinical and biological significance of immune markers, especially those reflecting the activation status of lymphocytes, should be performed on whole tissue sections, reducing sampling bias due to tumor heterogeneity, and providing a more comprehensive understanding of the complex tumor- immune dynamics. Moreover, we found that the expression of both PD-1 and LAG-3 highly correlated with the presence of TILs, especially cytotoxic CD8+ cells.

Even though stratified analysis according to levels of lymphocytic infiltration was not performed due to the low number of cases in each subgroup, our results suggest that the presence of PD-1+ and LAG-3+ TILs in the tumor microenvironment may reflect the occurrence of an active, although partially exhausted, intratumoral immune response, rather than representing a global marker of immunosuppression. Accordingly, emerging evidence have revealed that local immunomodulatory factors (e.g. IFN- $\gamma$ released by TILs), or the activation of oncogenic signaling pathways (e.g. the PI3K pathway) can promote the expression of PD-L1, which has been shown to be enriched in triple-negative/basal-like breast cancer, and to be associated with good outcome and response to chemotherapy in patients with TNBC [6, 10-14].

Interestingly, by analyzing two independent cohorts, we found that PD-1+ and LAG-3+ TILs were concurrently expressed in approximately $15 \%$ of TNBC cases. Thus, reversing the phenotype of exhausted $\mathrm{T}$ lymphocytes by targeting multiple inhibitory receptors may boost an effective anti-tumor immune response, and represent a novel valuable strategy to treat a subgroup of patients with TNBC. Recently, the blockade of the PD-1/ PD-L1 pathway has shown promising clinical activity in patients with metastatic TNBC, although molecular preselection of the candidate patients for novel clinical trials would be valuable [33, 35-38]. Preclinical data demonstrates that anti-LAG-3 is mildly effective as monotherapy, but potently synergizes with anti-PD-1, suggesting that the combined immune checkpoint inhibition could enhance $\mathrm{T}$ cell activity and improve antitumor immunity [32]. Furthermore, the dual blockade of PD-1 and LAG-3 may exhibit less immune toxicity than that observed with the blockade of other immune receptors (e.g. CTLA-4).

Even though we confirmed the prognostic value of TILs in TNBC, the biological link between FOXP3+ and $\mathrm{CD} 8+$ lymphocytes, and the clinical relevance of checkpoint receptors in patients with TNBC with different levels of TILs, warrant further investigations. Despite these potential limitations, our findings support the clinical evaluation of combination immunotherapies with anti-PD-1/PD-L1 and anti-LAG-3 in a specific subset of patients with TNBC who have concurrent expression of both checkpoints.

\section{Conclusions}

The presence of elevated TILs positively correlated with the density of all main $\mathrm{T}$ cell subtypes. The assessment of single immune components does not significantly improve risk stratification given by increasing stromal 
TILs, which remains an independent prognostic marker for TNBC treated with adjuvant chemotherapy containing anthracyclines. We have further demonstrated that the immune checkpoints PD-1 and LAG-3 are concurrently expressed in nearly $15 \%$ of tumors. The expression of PD-1 and LAG-3 is highly correlated with the presence of TILs, especially cytotoxic CD8+ cells, reflecting the occurrence of an effective intratumoral immune response. This study highlights the importance of different lymphocyte subpopulations for the selection of patients with primary TNBC who may benefit from immunomodulatory drugs. Our data support a clinical evaluation of anti-PD-1/PD-L1 and LAG-3 in combination with chemotherapy in a specific subset of patients with TNBC.

\section{Additional files}

Additional file 1: Table S1. Patient characteristics. Table S2. Antibodies and immunostaining protocols. Table S3. Associations between tumorinfiltrating lymphocytes and clinicopathological features in triple-negative breast cancer $(n=259)$. Table S4. Associations between tumor-infiltrating lymphocytes and clinicopathological features in triple-negative breast cancer (validation cohort; $n=104$ ). Table S5. Univariate Cox regression analysis of FOXP3 for relapse-free survival and overall survival in triplenegative breast cancer $(n=259)$ stratified by CD8+ tumor-infiltrating lymphocytes status. (PDF $90 \mathrm{~kb}$ )

Additional file 2: Figure S1. Prognostic value of the binary tumorinfiltrating lymphocytes (TILS) cutoff $\geq 20 \%$ in triple-negative breast cancer (TNBC) patients of the discovery cohort stratified by nodal status. Figure S2. Correlation between the expression of PD-1 and LAG-3 and the presence of CD8+ cells in triple-negative breast cancer (TNBC). (PDF $397 \mathrm{~kb}$ )

\section{Abbreviations}

Cl: confidence interval; CTLA-4: cytotoxic T-lymphocyte associated protein 4; FFPE: formalin-fixed, paraffin-embedded; FOXP3: forkhead box protein 3; HE: hematoxylin and eosin; HR: hazard ratio; IHC: immunohistochemistry; LAG-3: lymphocyte activation gene 3; LPBC: lymphocyte-predominant breast cancer; OS: overall survival; PBS: phosphate-buffered saline; PD-

1: programmed cell death 1; PD-L1: programmed cell death ligand 1; RFS: relapse-free survival; TILs: tumor-infiltrating lymphocytes; TNBC: triplenegative breast cancer

\section{Acknowledgments}

The authors are grateful to all colleagues working at the Breast Unit at Humanitas Clinical and Research Institute (Rozzano - Milan, Italy), and Humanitas Oncology Center of Catania (Catania, Italy) and Humanitas Mater Domini (Castellanza, Italy).

\section{Funding}

This study was supported by a grant from Associazione Italiana Ricerca sul Cancro (AIRC Grant 6251 to L. Santarpia); and Fondazione Italiana Ricerca sul Cancro (FIRC fellowship 18328 to G. Bottai). The work of S. Loi is supported by the Cancer Council Victoria, the National Breast Cancer Foundation Australia, the Breast Cancer Foundation, NY, USA.

\section{Availability of supporting data}

Not applicable.

\section{Authors' contributions}

LS conceived, designed, and supervised the study. GB, CR, and LS developed the methodology. GB, LDT, SL, AL, CR, JSRF, MR, LS, CS, CT, and RT participated in data acquisition. GB, LDT, SL, MR, and LS participated in data analysis and interpretation. GB, AM, CR, LS, and AS provided administrative, technical, or material support. All authors were involved in writing and reviewing the manuscript, and approved the final manuscript.

\section{Authors' information}

Not applicable.

\section{Competing interests}

The authors declare that they have no competing interests.

\section{Consent for publication}

Not applicable.

Ethics approval and consent to participate

The study was approved by the ethical committee of the Humanitas Hospitals.

\section{Author details}

${ }^{1}$ Oncology Experimental Therapeutics, IRCCS Clinical and Research Institute Humanitas, Via Manzoni 113, 20089 Rozzano-Milan, Italy. ${ }^{2}$ Department of Oncology, IRCCS Clinical and Research Institute Humanitas, Rozzano-Milan, Italy. ${ }^{3}$ Department of Pathology, IRCCS Clinical and Research Institute Humanitas, Rozzano-Milan, Italy. ${ }^{4}$ Department of Surgery, IRCCS Clinical and Research Institute Humanitas, Rozzano-Milan, Italy. ${ }^{5}$ Department of Pathology, Memorial Sloan Kettering Cancer Center, New York, NY, USA. ${ }^{6}$ Humanitas University, Rozzano-Milan, Italy. ${ }^{7}$ Breast Cancer Translational Research Laboratory, Institut Jules Bordet, Université Libre de Bruxelles, Brussels, Belgium. ${ }^{8}$ Department of Immunology and Inflammation, IRCCS Clinical and Research Institute Humanitas, Rozzano-Milan, Italy. ${ }^{9}$ Division of Cancer Medicine and Research, Peter MacCallum Cancer Centre, East Melbourne, Victoria, Australia.

Received: 28 April 2016 Accepted: 22 November 2016 Published online: 03 December 2016

\section{References}

1. Gluz O, Liedtke C, Gottschalk N, Pusztai L, Nitz U, Harbeck N. Triple-negative breast cancer-current status and future directions. Ann Oncol. 2009;20:1913-27.

2. Fridman WH, Pagès F, Sautès-Fridman C, Galon J. The immune contexture in human tumours: impact on clinical outcome. Nat Rev Cancer. 2012;12:298-306.

3. Adams S, Gray RJ, Demaria S, Goldstein L, Perez EA, Shulman LN, et al. Prognostic value of tumor-infiltrating lymphocytes in triple-negative breast cancers from two phase III randomized adjuvant breast cancer trials: ECOG 2197 and ECOG 1199. J Clin Oncol. 2014;32:2959-66.

4. Adams S, Goldstein LJ, Sparano JA, Demaria S, Badve SS. Tumor infiltrating lymphocytes (TILs) improve prognosis in patients with triple negative breast cancer (TNBC). Oncoimmunology. 2015;4, e985930.

5. Loi S, Sirtaine N, Piette F, Salgado R, Viale G, Van Eenoo F, et al. Prognostic and predictive value of tumor-infiltrating lymphocytes in a phase III randomized adjuvant breast cancer trial in node-positive breast cancer comparing the addition of docetaxel to doxorubicin with doxorubicinbased chemotherapy: BIG 02-98. J Clin Oncol. 2013;31:860-7.

6. Denkert C, von Minckwitz G, Brase JC, Sinn BV, Gade S, Kronenwett R, et al. Tumor-infiltrating lymphocytes and response to neoadjuvant chemotherapy with or without carboplatin in human epidermal growth factor receptor 2positive and triple-negative primary breast cancers. J Clin Oncol. 2015;33:983-91.

7. Loi S, Michiels S, Salgado R, Sirtaine N, Jose V, Fumagalli D, et al. Tumor infiltrating lymphocytes are prognostic in triple negative breast cancer and predictive for trastuzumab benefit in early breast cancer: results from the FinHER trial. Ann Oncol. 2014;25:1544-50.

8. Dushyanthen S, Savas P, Willard-Gallo K, Denkert C, Salgado R, Loi S. Tumour-infiltrating lymphocytes (TILs) in breast cancer: a predictive or a prognostic marker? Curr Breast Cancer Rep. 2015;7:59-70.

9. Nguyen LT, Ohashi PS. Clinical blockade of PD1 and LAG3 - potential mechanisms of action. Nat Rev Immunol. 2015;15:45-56.

10. Mittendorf EA, Philips AV, Meric-Bernstam F, Qiao N, Wu Y, Harrington S, et al. PD-L1 expression in triple-negative breast cancer. Cancer Immunol Res. 2014;2:361-70.

11. Gatalica Z, Snyder C, Maney T, Ghazalpour A, Holterman DA, Xiao N, et al. Programmed cell death 1 (PD-1) and its ligand (PD-L1) in common cancers 
and their correlation with molecular cancer type. Cancer Epidemiol Biomarkers Prev. 2014:23:2965-70.

12. Ali HR, Glont SE, Blows FM, Provenzano E, Dawson SJ, Liu B, et al. PD-L1 protein expression in breast cancer is rare, enriched in basal-like tumours and associated with infiltrating lymphocytes. Ann Oncol. 2015;26:1488-93.

13. Wimberly $H$, Brown JR, Schalper K, Haack H, Silver MR, Nixon C, et al. PD-L1 expression correlates with tumor-infiltrating lymphocytes and response to neoadjuvant chemotherapy in breast cancer. Cancer Immunol Res. 2015;3:326-32.

14. Schalper KA, Velcheti V, Carvajal D, Wimberly H, Brown J, Pusztai L, et al. In situ tumor PD-L1 mRNA expression is associated with increased TILs and better outcome in breast carcinomas. Clin Cancer Res. 2014;20:2773-82.

15. McShane LM, Altman DG, Sauerbrei W, Taube SE, Gion M, Clark GM. Reporting recommendations for tumor marker prognostic studies (REMARK). J Natl Cancer Inst. 2005;97:1180-4.

16. Salgado R, Denkert C, Demaria S, Sirtaine N, Klauschen F, Pruneri G, et al. The evaluation of tumor-infiltrating lymphocytes (TILs) in breast cancer: recommendations by an International TILs Working Group 2014. Ann Oncol. 2015;26:259-71.

17. Loi S, Drubay D, Adams S, Francis PA, Joensuu H, Dieci MV, et al. Pooled individual patient data analysis of stromal tumor infiltrating lymphocytes in primary triple negative breast cancer treated with anthracycline-based chemotherapy. [abstract]. In: Proceedings of the Thirty-Eighth Annual CTRCAACR San Antonio Breast Cancer Symposium: 2015 Dec 8-12; San Antonio, TX. Philadelphia (PA): AACR; Cancer Res 2016;76(4 Suppl):Abstract nr S1-03

18. Bordeaux J, Welsh A, Agarwal S, Killiam E, Baquero M, Hanna J, et al. Antibody validation. Biotechniques. 2010;48:197-209.

19. Fitzgibbons PL, Bradley LA, Fatheree LA, Alsabeh R, Fulton RS, Goldsmith JD, et al. Principles of analytic validation of immunohistochemical assays: guideline from the College of American Pathologists Pathology and Laboratory Quality Center. Arch Pathol Lab Med. 2014;138:1432-43.

20. Vassilakopoulou M, Parisi F, Siddiqui S, England AM, Zarella ER, Anagnostou $V$, et al. Preanalytical variables and phosphoepitope expression in FFPE tissue: quantitative epitope assessment after variable cold ischemic time. Lab Invest. 2015;95:334-41.

21. Llosa NJ, Cruise M, Tam A, Wicks EC, Hechenbleikner EM, Taube JM, et al. The vigorous immune microenvironment of microsatellite instable colon cancer is balanced by multiple counter-inhibitory checkpoints. Cancer Discov. 2015:5:43-51.

22. Taube JM, Young GD, McMiller TL, Chen S, Salas JT, Pritchard TS, et al. Differential expression of immune-regulatory genes associated with PD-L1 display in melanoma: implications for PD-1 pathway blockade. Clin Cancer Res. 2015:21:3969-76.

23. Mahmoud SM, Paish EC, Powe DG, Macmillan RD, Grainge MJ, Lee AH, et al. Tumor-infiltrating CD8+ lymphocytes predict clinical outcome in breast cancer. J Clin Oncol. 2011;29:1949-55.

24. Ali HR, Provenzano E, Dawson SJ, Blows FM, Liu B, Shah M, et al. Association between CD8+ T-cell infiltration and breast cancer survival in 12,439 patients. Ann Oncol. 2014;25:1536-43.

25. Liu S, Foulkes WD, Leung S, Gao D, Lau S, Kos Z, et al. Prognostic significance of FOXP3+ tumor-infiltrating lymphocytes in breast cancer depends on estrogen receptor and human epidermal growth factor receptor-2 expression status and concurrent cytotoxic T-cell infiltration. Breast Cancer Res. 2014;16:432

26. West NR, Kost SE, Martin SD, Milne K, Deleeuw RJ, Nelson BH, et al. Tumourinfiltrating FOXP3(+) lymphocytes are associated with cytotoxic immune responses and good clinical outcome in oestrogen receptor-negative breast cancer. Br J Cancer. 2013;108:155-62.

27. Jiang D, Gao Z, Cai Z, Wang M, He J. Clinicopathological and prognostic significance of FOXP3+ tumor infiltrating lymphocytes in patients with breast cancer: a meta-analysis. BMC Cancer. 2015;15:727.

28. Loi S. Host antitumor immunity plays a role in the survival of patients with newly diagnosed triple-negative breast cancer. J Clin Oncol. 2014;32:2935-7.

29. Tsoutsou PG, Bourhis J, Coukos G. Tumor-infiltrating lymphocytes in triplenegative breast cancer: a biomarker for use beyond prognosis? J Clin Oncol. 2015;33:1297-8.

30. Tung NM, Winer EP. Tumor-infiltrating lymphocytes and response to platinum in triple-negative breast cancer. J Clin Oncol. 2015;33:969-71.

31. Muenst S, Soysal SD, Gao F, Obermann EC, Oertli D, Gillanders WE. The presence of programmed death 1 (PD-1)-positive tumor-infiltrating lymphocytes is associated with poor prognosis in human breast cancer. Breast Cancer Res Treat. 2013;139:667-76.
32. Woo SR, Turnis ME, Goldberg MV, Bankoti J, Selby M, Nirschl CJ, et al. Immune inhibitory molecules LAG-3 and PD-1 synergistically regulate T-cell function to promote tumoral immune escape. Cancer Res. 2012;72:917-27.

33. Savas P, Salgado R, Denkert C, Sotiriou C, Darcy PK, Smyth MJ, et al. Clinical relevance of host immunity in breast cancer: from TILs to the clinic. Nat Rev Clin Oncol. 2016;13:228-41.

34. Okazaki T, Chikuma S, Iwai Y, Fagarasan S, Honjo T. A rheostat for immune responses: the unique properties of PD-1 and their advantages for clinical application. Nat Immunol. 2013;14:1212-8.

35. Gibson J. Anti-PD-L1 for metastatic triple-negative breast cancer. Lancet Oncol. 2015;16, e264.

36. Emens LA, Braiteh FS, Cassier P, Delord J-P, Eder JP, Fasso M, et al. inhibition of PD-L1 by MPDL3280A leads to clinical activity in patients with metastatic triple-negative breast cancer (TNBC). [abstract]. In: Proceedings of the 106th Annual Meeting of the American Association for Cancer Research; 2015 Apr 18-22; Philadelphia, PA. Philadelphia (PA): AACR; Cancer Res 2015;75(15 Suppl):Abstract nr 2859

37. Dirix LY, Takacs I, Nikolinakos P, Jerusalem G, Arkenau H-T, Hamilton EP, et al. Avelumab (MSB0010718C), an anti-PD-L1 antibody, in patients with locally advanced or metastatic breast cancer: A phase Ib JAVELIN solid tumor trial. [abstract]. In: Proceedings of the Thirty-Eighth Annual CTRCAACR San Antonio Breast Cancer Symposium: 2015 Dec 8-12; San Antonio, TX. Philadelphia (PA): AACR; Cancer Res 2016;76(4 Suppl):Abstract nr S1-04.

38. Adams S, Diamond J, Hamilton E, Pohlmann P, Tolaney S, Molinero L, et al. Safety and clinical activity of atezolizumab (anti-PDL1) in combination with nab-paclitaxel in patients with metastatic triple-negative breast cancer. [abstract]. In: Proceedings of the Thirty-Eighth Annual CTRC-AACR San Antonio Breast Cancer Symposium: 2015 Dec 8-12; San Antonio, TX. Philadelphia (PA): AACR; Cancer Res 2016;76(4 Suppl):Abstract nr P2-11-06.

\section{Submit your next manuscript to BioMed Central and we will help you at every step:}

- We accept pre-submission inquiries

- Our selector tool helps you to find the most relevant journal

- We provide round the clock customer support

- Convenient online submission

- Thorough peer review

- Inclusion in PubMed and all major indexing services

- Maximum visibility for your research

Submit your manuscript at www.biomedcentral.com/submit
) Biomed Central 